M. Yasugi

Nagoya Math. J.

Vol. 85"(1982), 175-188

\title{
CONTINUOUS VALUATION AND LOGIC
}

\author{
MARIKO YASUGI
}

We consider the continuous valuation of logic, where the certainty of a statement is measured with a number in the closed unit interval $I=[0,1]$.

The idea originates in continuous logics, which have been investigated from various standpoints, in [1] [4] and [8] [10], for example. More comprehensive information can be seen in [4] and others.

One of the problems which arises in studying continuous logics is the interpretation of the implication; the value of $A \rightarrow B$ is usually defined to be $1-([A]-[B])$, where $[A]$ represents the value of $A$. With this interpretation, however, the equality axiom can be dealt with only when the premisses are certain. This situation makes it infeasible to develop set theory along this line.

When working on various valuations, such as Boolean, Heyting and continuous, it has been a traditional practice to consider a logical system which is consistent (and preferably complete) with regards to a given valuation, and to investigate the models of the valid formulas, namely of the formulas which assume constantly the maximal element of the values.

It is interesting, however, to speculate on some theories which abide with the law of classical logic, while allowing deviant valuations. Also, it is natural for us to wish to work on the statements (or events) which are not necessarily valid in the sense state above; namely, we wish to study the situation where the value of a formula is $p$ for any $p$, reading it as "the degree of certainty of the statement is $p$ ", or "the statement is true with certainty $p$ ".

More specifically, we work in classical logics, first order and second order, hence in particular $A \rightarrow B$ is interpreted to be $7 A \vee B$. The value

Received November 30, 1979.

Revised August 5, 1980.

The author owes the revision of a part of this article to the referee. 
of $A$, which is temporarily denoted by $[A]$ and is assumed to be in the interval $I$, may be interpreted as indicating the degree of certainty of $A$ to occur or to hold.

The intended meaning of the logical connectives relative to our valuation is this: $[A \wedge B]$ is the degree of certainty with which one can predict the occurrence of $A$ and that of $B ;[A \vee B]$ is the degree of certainty with which one can predict whether $A$ occurs or $B$ occurs; $[7 A]$ is the reciprocal of $A$. Thus, $[A \wedge B]=\min ([A],[B]),[A \vee B]=\max ([A],[B])$, $[7 A]=1-[A]$, and $\forall$ and $\exists$ are regarded as generalizations of $\wedge$ and $\checkmark$ respectively (cf. $\S 1$ ).

If we say " $A$ is more certain than not" when $[A] \geq 1 / 2$, it turns out that provable formulas (in classical logics) are exactly those which are "more certain than not" (§§ 2-3).

As a somewhat restricted notion, we propose $p$-validity for $p>1 / 2$, and show the relative consistency of $p$-valid formulas with classical (second order) logic (§ 4).

The degree of certainty has no set theoretical interpretation, nor is it logic nor is it any physical quantity, but it is a way of thinking, or a way of conception of a certain kind of "liklihood" measured in the unit interval. Thus, for example, $[A \vee>A]=p$ (which is not necessarily 1) means that the certainty of whether $A$ occurs or its reciprocal occurs is $p$; this can be rephrased as that $A$ is determinate to the extent of $p$. $[A \wedge>A]=p$ (which is not necessarily 0 ) means that the certainty that $A$ occurs and its reciprocal occurs is $p$; here $p$ may be regareded as the degree of indeterminateness of $A$.

For the first order cases, similar ideas have been developed by some people working on fuzzy sets and logics and some results similar to ours are seen in some of their works; see [5] [7], [12] and [13].

As a sequel to this paper, we are planning an article in which $p$ validity $(p>1 / 2)$ of set theory will be demonstrated.

\section{$\S 1$. Valuation of first order language}

Let $I$ denote the closed unit interval of reals, let $D$ denote a nonempty set, which will serve as the domain of individuals, and let $F_{0}$ denote the family of functions whose domain is $D$ and whose range is $I$. Those will be fixed throughout, and represent the absolute world.

Definition 1.1. Let $L$ be any first order language of the predicate 
calculus, where the standard symbolism is assumed. Although it is irrelevant which formulation of the first order predicate calculus is assumed, we employ the Gentzen-type system, in which free variables and bound variables are distinguished (cf. Chapter I of [11]).

Let $\phi_{1}$ be a map from individual constants of $L$ to $D$, let $\phi_{2}$ be a map from function symbols to functions of the same arities whose arguments and values assume the elements in $D$, and let $\phi_{3}$ be a map from predicate symbols to functions in $F_{0}$ of the same arities. In the subsequent definition, $\phi_{1}, \phi_{2}$ and $\phi_{3}$ will be held fixed. Let $\psi$ be an assignment of an element. of $D$ to each free variable.

The valuation of $L$-terms and $L$-formulas $Z$ with regards to $D, \phi_{1}, \phi_{2}, \phi_{3}$ and $\psi$, denoted by $v\left(Z ; D, \phi_{1}, \phi_{2}, \phi_{3}, \psi\right)$, is defined below, where some or all of $D, \phi_{1}, \phi_{2}, \phi_{3}$, and $\psi$ will be spared in the expressions except when they need to be explicitly expressed.

$$
\begin{aligned}
& v(c ; \psi)=\phi_{1}(c) \text { if } c \text { is an individual constant. } \\
& v(a ; \psi)=\psi(a) \text { if } a \text { is a free variable. } \\
& v\left(f\left(t_{1}, \cdots, t_{m}\right) ; \psi\right)=\phi_{2}(f)\left(v\left(t_{1} ; \psi\right), \cdots, v\left(t_{m}, \psi\right)\right)
\end{aligned}
$$

if $f$ is a function symbol and $t_{1}, \cdots, t_{m}$ are terms.

$$
v\left(P\left(t_{1}, \cdots, t_{m}\right) ; \psi\right)=\phi_{3}(P)\left(v\left(t_{1} ; \psi\right), \cdots, v\left(t_{m} ; \psi\right)\right)
$$

if $P$ is a predicate symbol and $t_{1}, \cdots, t_{m}$ are terms.

$$
\begin{aligned}
& v(A \wedge B ; \psi)=\min (v(A ; \psi), v(B ; \psi)) . \\
& v(A \vee B ; \psi)=\max (v(A ; \psi), v(B ; \psi)) .
\end{aligned}
$$$$
v(\supset A ; \psi)=1-v(A ; \psi) \text {. }
$$$$
v(A \supset B ; \psi)=\max (1-v(A ; \psi), v(B ; \psi)) \text {. }
$$

$v(\forall x A(x) ; \psi)=\inf \{v(A(a) ; \psi[a / d]) ; d \in D\}$, where $a$ is the first free variable (in some predetermined order) not occurring in $A(x)$ and $\psi[a / d]$ stands for the assignment obtained from $\psi$ by assigning $d$ to $a$.

$$
v(\exists x A(x) ; \psi)=\sup \{v(A(a) ; \psi[a / d]) ; d \in D\} .
$$

Proposition 1.1. 1) For any terms $s$ and $t, v(s ; \psi)=v(t ; \psi)$ implies $v(A(s) ; \psi)=v(A(t) ; \psi)$ for every $A$.

2) If a free variable a does not occur in $A(s)$ and if $v(s)=d$, then $v(A(a) ; \psi[a / d])=v(A(s) ; \psi)$.

Proposition 1.2. 1) $v(A \supset A)=v(A \vee>A) \quad(=v(A)$ if $v(A) \geq 1 / 2$; $=1-v(A)$ otherwise). Thus, $v(A \vee>A)=v(A \supset A) \geq 1 / 2$, but the value is not necessarily 1. In fact, the value is 1 if and only if $v(A)=1$ or 0 , that is, when $A$ is determinate, so to speak. 
2) $v(A \wedge>A)=1-v(A)$ if $v(A) \geq 1 / 2 ;=v(A)$ otherwise. Thus, $v(A \wedge>A) \leq 1 / 2$, and the value is 0 when $A$ is determinate.

3) For any propositional function $\pi$ which involves $A$ alone,

$$
v(A \wedge>A) \leq v(\pi(A)) \leq v(A \vee>A) .
$$

Proposition 1.3. 1) $v(A) \leq v(B)$ implies $v(A \supset B) \geq 1 / 2$, but not necessarily the converse. The latter means that $v(A \supset B) \geq 1 / 2$ does not define any order relation between $A$ and $B$.

2) $v(A \supset B)=1$ if and only if $v(A)=0$ or $v(B)=1 ;=0$ if and only if both $v(A)=1$ and $v(B)=0$ hold.

3) If $v(A \supset B) \geq 1 / 2$ and $v(A) \geq 1 / 2$, then $v(B) \geq 1 / 2$.

Proposition 1.4. 1) The logical connectives can be expressed in terms of other ones; for example, $v(A \vee B)=v(>(>A \vee>B))$ and $v(\exists x A(x))=$ $v(>\forall x>A(x))$.

2) The value of a formula by $v$ is equal to that of its prenex normal form.

It is a routine work to establish this; try, for instance, $v(A \vee \exists x B(x))$ $=v(\exists x(A \vee B(x)))$.

Definition 1.2. Let $L\{=\}$ be the language $L$ augmented by $=$. = will be given the standard valuation, viz.,

$$
v(a=b)= \begin{cases}1 & \text { if } v(a)=v(b) \text { in } D, \\ 0 & \text { otherwise } .\end{cases}
$$

Note. The symbol $=$ will be used in various different contexts, but the distinctions should be made out easily.

Remark. What holds for $L$ holds for $L\{=\}$ as well.

Definition 1.3. For a fixed valuation $v$, we define some concepts with regards to $v$.

$v(A)$ is interpreted as expressing the degree of certainty of $A$; thus $v(A)=p$ is understood to mean $A$ is certain (certainly true, or $A$ is certain to occur) to degree $p$.

When $v(A) \geq 1 / 2$, we say $A$ is more certain (than not).

$\delta(A)=v(A \vee>A)$; this will be called the degree of definitiveness of $A$.

$\nu(A)=v(A \wedge>A)$; this will be called the degree of non-definitiveness of $A$. 
When $\delta(A)>1 / 2$, we say that $A$ is definitive; when $\delta(A)=1 / 2$, we say $A$ is non-definitive.

When $\delta(A) \geq p$, we say $A$ is definitive to degree $p$, or $A$ is $p$-definitive.

Corollary. $\quad \delta(A) \geq 1 / 2$ and $\nu(A) \leq 1 / 2$. Thus, $p$-definitiveness can be significant only when $p>1 / 2$.

\section{§2. Valuation and logic}

We consider either $L$ or $L\{=\}$.

LEMma 2.1. Let $G_{0}$ denote the set of functions from $D$ to $\left\{t_{r}, f_{s}\right\}$, where $t_{r}$ stands for truth and $f_{s}$ stands for falsehood. Let $\phi_{1}, \phi_{2}, \phi_{3}, \psi$ and $v$ be as before. Let $\chi$ denote a map from predicate symbols to functions in $G_{0}$ of the same arities, and let $w$ denote the classical two valued interpretation of logic based on $\phi_{1}, \phi_{2}, \chi$ and $\psi$. For any fixed $D, \phi_{1}, \phi_{2}$, the following hold.

1) For every $\chi$, there exists $a \phi_{3}$ such that

$$
w(A ; \chi, \psi)=t_{r}\left(f_{s}\right) \quad \text { if and only if } v\left(A ; \phi_{3}, \psi\right)=1(0),
$$

for every formula $A$ and every assignment $\psi$.

2) For every $\phi_{3}$, there exists a $\chi$ such that

$$
v\left(A ; \phi_{3}, \psi\right) \geq 1 / 2 \quad \text { if } w(A ; \chi, \psi)=t_{r},
$$

and

$$
v\left(A ; \phi_{3}, \psi\right) \leq 1 / 2 \quad \text { if } w(A ; \chi, \psi)=f_{s} .
$$

Notice that in 2) only the necessary condition is required of $w$.

The lemma can be easily established by identifying $t_{r}$ with 1 and $f_{s}$ with 0 in case of 1 ), and by collapsing $\geq 1 / 2$ to $t_{r}$ and $<1 / 2$ to $f_{s}$ in case of 2).

Theorem 1. A formula $A$ is logically valid if and only if $v\left(A ; D, \phi_{1}\right.$, $\left.\phi_{2}, \phi_{3}, \psi\right) \geq 1 / 2$ for every $D, \phi_{1}, \phi_{2}, \phi_{3}$ and $\psi$.

The theorem is an immediate consequence of Lemma 2.1.

Theorem 2. A formula $A$ is provable (in the first order predicate calculus with or without equality axioms) if and only if $v\left(A ; D, \phi_{1}, \phi_{2}, \phi_{3}, \psi\right)$ $\geq 1 / 2$ for every $D, \phi_{1}, \phi_{2}, \phi_{3}, \psi$.

This follows from Theorem 1 above and the completeness theorem. 


\section{§3. Valuation and second order logic}

There have been various speculations on second order axioms and set theory based on continuous logics, among them works by C. C. Chang [1] [3] and Takahashi [10]; detailed references are seen in C. C. Chang and Keisler [4]. In our case, however, logic is classical, hence the implication plays no particular role, and our concern lies on the statements which are more certain than not (cf. Definition 1.3). The comprehension causes no problem here.

Definition 3.1. Let $L^{\prime}$ be any standard second order language, where second order free variables will be denoted by $\alpha, \beta, \cdots$ and bound ones by $X, Y, \cdots$. To simplify the matter, we exclude constants unless otherwise required.

Definition 3.2. Let $D$ denote a domain as in $\S 1$.

1) Let $G$ denote any subset of $G_{0}$ (cf. Lemma 2.1), and define $H$ to be the pair $(D, G)$, which will serve as a structure for $L^{\prime}$. Let $\psi$ be as in $\S 1$ and let $\sigma$ denote an assignment of elements of $G$ of appropriate arities to second order free variables. $i(A ; H, \psi, \sigma)$ will represent the Henkin-type interpretation of $A$ with regards to $H, \psi$ and $\sigma, A$ an $L^{\prime}$ formula. For an abstract $V \equiv\left\{x_{1}, \cdots, x_{n}\right\} A\left(x_{1}, \cdots, x_{n}\right)$

$$
i(V ; H, \psi, \sigma)=g \text {, }
$$

where

$$
g\left(d_{1}, \cdots, d_{n}\right)=i\left(A\left(a_{1}, \cdots, a_{n}\right) ; H, \psi\left[a_{1} / d_{1}\right] \cdots\left[a_{n} / d_{n}\right], \sigma\right)
$$

for every $d_{1}, \cdots, d_{n}$ in $D$, where $a_{1}, \cdots, a_{n}$ are distinct free variables not occurring in $V$. A structure $H$ is called a Henkin structure if for every pair of $\psi$ and $\sigma, i(V ; H, \psi, \sigma)$ belongs to $G$ for every $V$. (The notions defined above are second order cases of those seen in, for example, Definition 21.4 of [11].)

2) Let $F$ denote any subset of $F_{0}$ (cf. $\S 1$ ), and define $K$ to be $(D, F)$, which will serve as a continuous structure for $L^{\prime}$. Let $\psi$ be as before and let $\tau$ be a second order assignment in $F . u(A ; K, \psi, \tau)$ will represent the valuation of an $L^{\prime}$-formula $A$ with regards to $K, \psi$ and $\tau$, which is defined in the same manner as the $v$ in Definition 1.1 with some additional cases, which are listed below. $K$ will be omitted in most cases.

$$
u\left(\alpha\left(a_{1}, \cdots, a_{n}\right) ; \psi, \tau\right)=\tau(\alpha)\left(\psi\left(a_{1}\right), \cdots, \psi\left(a_{n}\right)\right) .
$$




$$
\begin{aligned}
& u(\forall X A(X) ; \psi, \tau)=\inf \{u(A(\alpha) ; \psi, \tau[\alpha / f]) ; f \in F\} . \\
& u(\exists X A(X) ; \psi, \tau)=\sup \{u(A(\alpha) ; \psi, \tau[\alpha / f]) ; f \in F\} .
\end{aligned}
$$

Here, $\alpha$ is a second order free variable which does not occur in $A(X)$, $\tau[\alpha / f]$ is the assignment obtained from $\tau$ by assigning $f$ to $\alpha$, and $f$ ranges over the functions in $F$ of the same arity as $X$.

$$
u\left(\left\{x_{1} \cdots x_{n}\right\} A\left(x_{1}, \cdots, x_{n}\right) ; \psi, \tau\right)=f,
$$

where

$$
f\left(d_{1}, \cdots, d_{n}\right)=u\left(A\left(a_{1}, \cdots, a_{n}\right) ; \psi\left[a_{1} / d_{1}\right] \cdots\left[a_{n} / d_{n}\right], \tau\right)
$$

for every $d_{1}, \cdots, d_{n}$ in $D$.

A continuous structure $K=(D, F)$ is called a continuous Henkin structure if for every $\psi, \tau, u(V ; K, \psi, \tau)$ belongs to $F$ for every $V$.

In the subsequent discussion, $H$ and $K$ will denote Henkin structures.

Lemma 3.1. 1) For every $H=(D, G)$ there exists a $K=(D, F)$ such that for every $\sigma$ there is a $\tau$ satisfying:

$$
i(A ; H, \psi, \sigma)=t_{r}\left(f_{s}\right) \text { if and only if } u(A ; K, \psi, \tau)=1(0) \text {, }
$$

and

$$
\begin{aligned}
& i(V ; H, \psi, \sigma)\left(d_{1}, \cdots, d_{n}\right)=t_{r}\left(f_{s}\right) \text { if and only if } \\
& u(V ; K, \psi, \tau)\left(d_{1}, \cdots, d_{n}\right)=1(0)
\end{aligned}
$$

for every $A, V$ and $\psi$.

2) Let $K$ be $(D, F)$. For each $f$ in $F$, we define:

$$
\begin{aligned}
\Gamma(f)=\{ & g \in G_{0} ; g \text { is of the same arity as } f \text { and, for each } \\
& d_{1}, \cdots, d_{n}, g\left(d_{1}, \cdots, d_{n}\right)=t_{r} \text { implies } \\
& f\left(d_{1}, \cdots, d_{n}\right) \geq 1 / 2 \text {, while } g\left(d_{1}, \cdots, d_{n}\right)=f_{s} \\
& \text { implies } \left.f\left(d_{1}, \cdots, d_{n}\right) \leq 1 / 2\right\} .
\end{aligned}
$$

Put $G=\bigcup\{\Gamma(f) ; f \in F\} . \quad G$ is a subset of $G_{0}$. Put $H=(D, G) ; H$ is a structure for $L^{\prime}$. We say $\sigma$ is in $\Gamma$-relation to $\tau$ if $\sigma(\alpha)$ is in $\Gamma(\tau(\alpha))$ for every $\alpha$.

Now, for every $\tau, A, V$ and $\psi$, and for every $\sigma$ in $\Gamma$-relation to $\tau$, the following hold.

$$
\begin{aligned}
& u(A ; K, \psi, \tau) \geq 1 / 2(\leq 1 / 2) \text { if } \\
& i(A ; H, \psi, \sigma)=t_{r}\left(f_{s}\right)
\end{aligned}
$$




$$
\begin{aligned}
& u(V ; K, \psi, \tau)\left(d_{1}, \cdots, d_{n}\right) \geq 1 / 2(\leq 1 / 2) \text { if } \\
& i(V ; H, \psi, \sigma)\left(d_{1}, \cdots, d_{n}\right)=t_{r}\left(f_{s}\right) .
\end{aligned}
$$

As a consequence, $H$ is a Henkin structure.

Notice that in 2) only the necessary condition is required of $u$.

Proof. Since 1) is trivial, we shall establish 2) by induction on the complexity of $A$. We always assume $\sigma$ is in $\Gamma$-relation to $\tau$.

Suppose $i(\forall X A(X) ; H, \psi, \sigma)=t_{r}$, viz., $i(A(\alpha) ; H, \psi, \sigma[\alpha / g])=t_{r}$ for every $g$ in $G$. For each $f$ in $F$, consider $\tau^{\prime} \equiv \tau[\alpha / f]$. For each $g$ in $\Gamma(f) \equiv$ $\Gamma\left(\tau^{\prime}(\alpha)\right), \sigma^{\prime} \equiv \sigma[\alpha / g]$ is in $\Gamma$-relation to $\tau^{\prime}$. So, by the induction hypotheses, $u\left(A(\alpha) ; K, \psi, \tau^{\prime}\right) \geq 1 / 2$ for every $f$, from which follows $u(\forall X A(X) ; K, \psi, \tau)$ $\geq 1 / 2$.

Suppose $i(\forall X A(X) ; H, \psi, \sigma)=f_{s}$, viz., $i(A(\alpha) ; H, \psi, \sigma[\alpha / g])=f_{s}$ for some $g$ in $G$. But $g \in \Gamma(f)$ for some $f$ in $F$, so for such an $f, \sigma^{\prime} \equiv \sigma[\alpha / g]$ is in $\Gamma$-relation to $\tau^{\prime} \equiv \tau[\alpha / f]$. Thus, by the induction hypothesis, $u(A(\alpha) ; K, \psi$, $\left.\tau^{\prime}\right) \leq 1 / 2$, from which follows $u(\forall X A(X) ; K, \psi, \tau) \leq 1 / 2$.

$\exists X A(X)$ can be dealt with in a reciprocal manner. Other cases cause no difficulty.

(1) $u\left(\left\{x_{1} \cdots x_{n}\right\} A\left(x_{1}, \cdots, x_{n}\right) ; K, \psi, \tau\right)\left(d_{1}, \cdots, d_{n}\right)$

$$
=u\left(A\left(a_{1}, \cdots, a_{n}\right) ; K, \psi\left[a_{1} / d_{1}\right] \cdots\left[a_{n} / d_{n}\right], \tau\right) .
$$

(2) $i\left(\left\{x_{1} \cdots x_{n}\right\} A\left(x_{1}, \cdots, x_{n}\right) ; H, \psi, \sigma\right)\left(d_{1}, \cdots, d_{n}\right)$

$$
=i\left(A\left(a_{1}, \cdots, a_{n}\right) ; H, \psi\left[a_{1} / d_{1}\right] \cdots\left[a_{n} / d_{n}\right], \sigma\right) \text {. }
$$

The right hand side of $(1)$ is $\geq 1 / 2(\leq 1 / 2)$ if that of $(2)=t_{r}\left(f_{s}\right)$, hence the same relation holds for the left hand side.

Consider $i(V ; H, \psi, \sigma)$ for an abstract $V$, where $\sigma$ is an arbitrary assignment in $H$. For each $\alpha$, if $\sigma(\alpha)=g$, then $g \in \Gamma(f)$ for some $f \in F$, so choose such an $f$ and denote it by $f_{\alpha}$. If we define $\tau(\alpha)=f_{\alpha}$, then $\alpha$ is in $\Gamma$-relation to $\tau$, thus by (1) and (2) above, $u(V ; K, \psi, \tau)\left(d_{1}, \cdots, d_{n}\right) \geq 1 / 2(\leq 1 / 2) \quad$ if $i(V ; H, \psi, \sigma)\left(d_{1}, \cdots, d_{n}\right)=t_{r}\left(f_{s}\right)$. $f_{0} \equiv u(V ; K, \psi, \tau)$ is a function in $F$ by assumption; if we let $g_{0}$ be $i(V ; H, \psi, \sigma)$, which is a function in $G_{0}, g_{0} \in \Gamma\left(f_{0}\right)$, hence $g_{0} \in G$. Thus, $H$ is a Henkin structure.

Theorem 3. A closed L'-formula is Henkin-valid if and only if it is "more certain than not" in continuous valuation, namely, in any continuous Henkin structure the valuation assumes a value $\geq 1 / 2$ for any assignment. 
Proof. First, notice that the validity (in any sense) of a closed formula depends solely on a structure (and on an interpretation of constants), thus is independent of assignments. Let $K$ be any continuous Henkin structure. By 2) of Lemma 3.1, we can define a Henkin structure $H$, which satisfies that if a closed formula assumes value $t_{r}$ in $H$, then its value in $K$ is $\geq 1 / 2$, which proves the "only if" part. The "if" part follows from 1) of Lemma 3.1.

Theorem 4. A closed L'-formula is provable in the second order predicate calculus (with the full comprehension) if and only if it is "more certain than not" in every continuous Henkin structure.

Proof. This is a consequence of Theorem 3 above and the Henkincompleteness (cf. $\S 21$ of [11], for example) of the second order logic.

Proposition 3.1. If $u(V ; K, \psi, \tau)=f$ and $\tau^{\prime} \equiv \tau[\alpha / f]$, then

$$
u(A(V) ; K, \psi, \tau)=u\left(A(\alpha) ; K, \psi, \tau^{\prime}\right)
$$

for every $A$.

Definition 3.3. All the notions in Definition 1.3 can be modified so that they become the notions with regards to $u$.

Let $\delta(A ; \psi)$ denote the $\delta(A)$ where the first order assignment is $\psi$.

$$
\begin{aligned}
& \delta\left(\left\{x_{1} \cdots x_{n}\right\} A\left(x_{1}, \cdots, x_{n}\right) ; \psi\right) \\
& \quad=\inf \left\{\delta\left(A\left(a_{1}, \cdots, a_{n}\right) ; \psi\left[a_{1} / d_{1}\right] \cdots\left[a_{n} / d_{n}\right]\right) ; d_{1}, \cdots, d_{n} \in D\right\} .
\end{aligned}
$$

CoRollary. $\delta(\mathrm{A}) \geq 1 / 2$ for $A$ an arbitrary formula or abstract.

Proposition 3.2. The definitions and results in this section can be easily extended to the cases where $L^{\prime}$ has various constants. A parallel to Proposition 1.1 holds for $L^{\prime}$ too.

\section{§4. Definitive valuation}

Here we shall study the valuations based on "definitive" assignments, namely on the set of functions whose values are bounded from below by a number greater than $1 / 2$. Since the treatments are quite similar in both cases, first order and second order, we shall mainly consider the second order case.

Definition 4.1. Let $f$ be in $F_{0}$. Define $\delta(f)$ : 


$$
\delta(f)=\inf \left\{\max \left(1-f\left(d_{1}, \cdots, d_{n}\right), f\left(d_{1}, \cdots, d_{n}\right)\right) ; d_{1}, \cdots, d_{n} \in D\right\} .
$$

If $\delta(f) \geq p$, we say $f$ is definitive to degree $p$ (p-definitive). Notice that $1 / 2 \leq \delta(f) \leq 1$.

A subset $F$ of $F_{0}$ is said to be a $p$-set if it consists of $p$-definitive functions alone. Let $K(p)$ be the family of structures $K=(D, F)$, where $F$ stands for a $p$-set. We consider second order valuations in the continuous Henkin structures which belong to $K(p)$. We say such a structure is $p$-definitive.

[Assumption] Henceforth $p>1 / 2$ will be assumed throughout.

Proposition 4.1. To economize the notation, we write $\Xi$ for $K=$ $(D, F), \phi_{1}, \phi_{2}, \phi_{3}$ when those are fixed, where $K$ is a p-definitive structure, and $\phi_{1}, \phi_{2}$, and $\phi_{3}$ are maps from respective constants to appropriate objects. Now, for any $\Xi$,

(1) either $u(A ; \Xi, \psi, \tau) \geq p$ or $u(A, \Xi, \psi, \tau) \leq 1-p$, and hence

(2) $\delta(A)=\delta(A ; \Xi, \psi, \tau) \geq p$

for every $A$ a formula or an abstract and for every assignments $\psi$ and $\tau$ (cf. Definitions 1.3 and 3.3).

Proof. By induction on the complexity of $A$.

A is $\alpha(a) . \quad u(A ; \psi, \tau)=f(d)$ for some $f$ in $F$ and $d$ in $D$. Since $K$ is $p$-definitive, $\max (1-f(d), f(d)) \geq p$, hence $f(d) \geq p$ or $f(d) \leq 1-p$.

$\mathrm{A}$ is $\forall X B(X) . \quad u(A ; \tau)=\inf \{u(B(\alpha) ; \tau[\alpha / f]) ; f \in F\}$. By the induction hypothesis, for each $f$, either $u(B(\alpha) ; \tau[\alpha / f]) \geq p$ or $u(B(\alpha) ; \tau[\alpha / f]) \leq 1-p$. If $\geq p$ holds for every $f$, then $u(A ; \tau) \geq p$ follows. If $\leq 1-p$ for some $f$, then $u(A ; \tau) \leq 1-p$ follows.

Other cases can be dealt with in a similar manner.

(2) follows immediately from (1).

Corollary. In any $p$-definitive structure, $u(A \vee>A)=u(A \supset A) \geq p$.

Definition 4.2. A valuation in a $p$-definitive structure is called a $p$-definitive valuation. We shall henceforth consider $p$-definitive valuations for a $p>1 / 2$.

Definition 4.3. Let $E$ be as above. A second order formula $A$ is said to be $p$-valid with regards to $\Xi$ if $u(A ; \Xi, \psi, \tau) \geq p$ for every $\psi$ and $\tau$ in $K$. 
THEOREM 5. Fix a $\Xi$. Let $\mathscr{A}$ be a set of formulas of $L^{\prime}\left(L^{\prime}\right.$ with or without constants), each of which is p-valid with regards to $\Xi$. (Let us call such an $\mathscr{A}$ a p-valid set with regards to $\Xi$.) Let $\mathscr{P}(\mathscr{A})$ be the second order predicate calculus augmented by the formulas of $\mathscr{A}$ as axioms. Then, any formula which is provable in $\mathscr{P}(\mathscr{A})$ is p-valid with regards to $\Xi$.

Proof. Although it is irrelevant which formulation of logic we take, we shall work with the Gentzen-type second order system, where the formulas of $\mathscr{A}$ are added as initial sequents and $\rightarrow$ is interpreted as $\supset$ (cf. $\S 15$ of [11]).

We prove the theorem by induction on the complexity of a formal proof in $\mathscr{P}(\mathscr{A})$.

For the axioms in $\mathscr{A}$, the $p$-validity is assumed.

$u(A \rightarrow A) \geq p$ by Corollary of Proposition 4.1.

Let $I$ be the last inference in a proof and assume the theorem for the upper sequent(s) of $I$.

$I$ is a first order $\forall$ left.

$$
I \frac{A(s), \Gamma \rightarrow \Delta}{\forall x A(x), \Gamma \rightarrow \Delta}
$$

To simplify the matter, we assume $\Gamma$ and $\Delta$ are respectively singular formulas.

(1) $u(\forall x A(x), \Gamma \rightarrow \Delta)=\max (1-u(\forall x A(x) ; \psi), 1-u(\Gamma ; \psi), u(\Delta ; \psi))$, where $1-u(\forall x A(x) ; \psi)=\sup \{1-u(A(a) ; \psi[a / d])) ; d \in D\}$.

If $(1)<p$, then $1-u(\Gamma ; \psi)<p, u(\Delta ; \psi)<p$ and $1-u(A(a) ; \psi[a / d])<p$ for every $d$ in $D$. In particular, this is so for $d \equiv u(s ; \psi)$. Then $u(A(a)$; $\psi[a / d])=u(A(s) ; \psi)(\mathrm{cf} .2)$ of Proposition 1.1), hence $1-u(A(s) ; \psi)<p$. But then $u(A(s), \Gamma \rightarrow \Delta ; \psi)<p$, contradicting the induction hypothesis.

$I$ is a second order left.

$$
I \frac{A(V), \Gamma \rightarrow \Delta}{\forall X A(X), \Gamma \rightarrow \Delta}
$$

(2) $u(\forall X A(X), \Gamma \rightarrow \Delta ; \psi, \tau)$

$$
=\max (1-u(\forall X A(X) ; \psi, \tau), 1-u(\Gamma ; \psi, \tau), u(\Delta ; \psi, \tau)) .
$$

If $(2)<p$, then $1-u(\Gamma ; \psi, \tau)<p, u(\Delta ; \psi, \tau)<p$ and $1-u(A(\alpha) ; \psi, \tau[\alpha / f])$ $<p$ for every $f$ in $F$. Let $f$ be $u(V ; \psi, \tau)$ and let $\tau^{\prime}$ be $\tau[\alpha / f]$. Then $u(A(V) ; \psi, \tau)=u\left(A(\alpha) ; \psi, \tau^{\prime}\right)$ (cf. Proposition 3.1). So $1-u(A(V) ; \psi, \tau)<p$, implying that the value of the upper sequent of $I$ is below $p$, yielding a contradiction. 
$I$ is a second order $\forall$ right.

$$
I \frac{\Gamma \rightarrow \Delta, A(\alpha)}{\Gamma \rightarrow \Delta, \forall X A(X)}
$$

3) $u(\Gamma \rightarrow \Delta, \forall X A(X) ; \tau)$

$$
=\max (1-u(\Gamma ; \tau), u(\Delta ; \tau), u(\forall X A(X) ; \tau)) .
$$

If $(3)<p$, then $1-u(\Gamma ; \tau)<p, u(\Delta ; \tau)<p$ and $u(A(\alpha) ; \tau[\alpha / f])<p$ for some $f$ in $F$. Put $\tau^{\prime} \equiv \tau[\alpha / f], u\left(\Gamma ; \tau^{\prime}\right)=u(\Gamma ; \tau)$ and $u\left(\Delta ; \tau^{\prime}\right)=u(\Delta ; \tau)$. So, $u\left(\Gamma \rightarrow \Delta, A(\alpha) ; \tau^{\prime}\right)<p$, contradicting the induction hypothesis.

$I$ is a cut.

$$
I \frac{\Gamma \rightarrow \Delta, D \quad D, \Pi \rightarrow \Lambda}{\Gamma, \Pi \rightarrow \Delta, \Lambda}
$$

Suppose the value of the lower sequent is below $p$. Then $1-u(\Gamma)$, $1-u(\Pi), u(\Delta), u(\Lambda)<p$. In order that the upper sequents assume values $\geq p$ under the same assignment, $u(D) \geq p$ and $1-u(D) \geq p$, but then $p$ $\leq 1 / 2$, contradicting the major assumption that $p>1 / 2$.

Other cases can be treated similarly.

Note. The argument above does not necessarily go through for $p=$ 1/2. (Examine the case where $I$ is a cut.)

Examples of $p$-valid sets $\mathscr{A}$.

$\mathscr{A} 1$. A consists of the equality axioms:

$$
s=t, \quad A(s) \longrightarrow A(t)
$$

for arbitrary $s, t$ and $A$, where the language is assumed to have $=$.

(*) $u(s=t, A(s) \rightarrow A(t))=\max (1-u(s=t), 1-u(A(s)), u(A(t)))$.

Case 1. $u(s=t)=1$, viz., $u(s)=u(t)$ in $D$ (cf. Definition 1.2). Then, by 1) of Proposition 1.1, $u(A(s))=u(A(t))$, so $(*)=\max (1-u(A(s))$, $u(A(s))) \geq p$.

Case 2. $u(s=t)=0 . \quad(*)=1 \geq p$.

$\mathscr{A}$. Consider the language of second order arithmetic with the constants $0,+1$ and $=$. Let $K$ be $(N, F)$, where $N$ is the set of natural numbers and $F$ is an arbitrary $p$-set. Let $\phi_{1}, \phi_{2}$ and $\phi_{3}$ be the natural interpretation of this language in $N$. Let $\mathscr{A}$ consist of the axioms of induction: 


$$
A(0), \quad \forall x(A(x) \supset A(x+1)) \longrightarrow \forall x A(x)
$$

for arbitrary $A$. Let $S$ stand for any sequent in $\mathscr{A}$.

$$
\begin{aligned}
u(S ; \psi)= & \max (1-u(A(0) ; \psi), \\
& 1-u(\forall x(A(x) \supset A(x+1)) ; \psi), \\
& u(\forall x A(x))),
\end{aligned}
$$

where the latter two terms are:

$$
\sup \{\min (u(A(a) ; \psi[a / n]), 1-u(A(a+1) ; \psi[a / n])) ; n \in N\}
$$

and

$$
\inf \{u(A(a) ; \psi[a / n]) ; n \in N\}
$$

respectively.

If $u(S ; \psi)<p$, then

(1) $1-u(A(0) ; \psi)<p$,

(2) $\min (u(A(a) ; \psi[a / n]), 1-u(A(a+1) ; \psi[a / n]))<p$

for every $n$, and

(3) $u(A(a) ; \psi[a / m])<p$

for some $m$ in $N$.

We shall establish, by (1) and (2), that, for each $n$,

(**) $u(A(a) ; \psi[a / n]) \geq p$.

This contradicts (3). So $u(S ; \psi) \geq p$. To simplify the notation, we write $u(A(n))$ for $u(A(a) ; \psi[a / n])$. Now the proof of $(* *)$ by induction on $n$.

$n=0 . \quad 1-u(A(0))<p$ by (1), viz., $u(A(0))>1-p$. But $u(A(0)) \geq p$ or $u(A(0)) \leq 1-p$ (Proposition 4.1), hence $u(A(0)) \geq p$.

$n+1$. Assume $(* *)$ for $n ; u(A(n)) \geq p$. Then, by $(2), 1-u((A(n+1))$ $<p$, or $u(A(n+1))>1-p$. But $u(A(n+1)) \geq p$ or $u(A(n+1)) \leq 1-p$. So $u(A(n+1)) \geq p$.

Note. $\mathscr{A} 1$ and $\mathscr{A} 2$ hold when $p=1 / 2$ just the same. We have placed the condition $p>1 / 2$ in order to comply with the theorem. (See the note after Theorem 5.)

Definition 4.4. A formula $A$ is said to be definitively valid if for every $p>1 / 2 A$ is $p$-valid with regards to every $p$-definitive valuation.

Theorem 6. A closed formula is definitively valid if and only if it is provable (in the second order predicate calculus), hence definitive validity is equivalent to Henkin-validity as well as continuous validity. 
Proof. A provable formula is definitively valid; this is a special case of Theorem 5 where $\mathscr{A}$ is empty. When $p=1, p$-validity is Henkin-validity. Those facts, Henkin-completeness and Theorems 3 and 4 in $\S 3$ establish the theorem.

Proposition 4.2. For every $u, A$ and $B, u(A \supset B) \geq p$ is equivalent to " $u(A) \geq p$ implies $u(B) \geq p$ ".

This is a corollary of Proposition 4.1.

Proposition 4.3. Let $\left(A_{\lambda}, \psi_{\lambda}\right)$ be a pair of a formula and an assignment indexed by $\lambda \in \Lambda$. Then $\sup \left\{u\left(A_{\lambda} ; \psi_{\lambda}\right) ; \lambda \in \Lambda\right\} \geq p$ if and only if $u\left(A_{\lambda}\right.$; $\left.\psi_{\lambda}\right) \geq p$ for some $\lambda \in \Lambda$. Thus, in particular, $u(\exists x A(x)) \geq p$ if and only if $u(A(a) ; \psi[a / d]) \geq p$ for some $d$ in $D$.

Proof. Use Proposition 4.1 and the fact that $1-p<p$.

\section{REFERENCES}

[1] C. C. Chang, Algebraic analysis of many valued logics, Trans. Amer. Math. Soc., 88 (1958), 467-490.

[2] - The axiom of comprehension in infinite valued logic, Math. Scand., 13 (1963), 9-30.

[ 3 ] - Infinite valued logic as a basis for set theory, Logic, Methodology and Philosophy of Science ed. by Y. Bar-Hillel (1965), 93-100.

[ 4 ] C. C. Chang and H. J. Keisler, Continuous Model Theory, Princeton University Press, Princeton, 1965.

[ 5 ] J. A. Goguen, L-fuzzy sets, J. Math. Anal. Appl., 18 (1967), 145-174.

[6] I. Grattan-Guiness, Fuzzy membership and many-valued quantities, Z. math. Log. Grundl. Math., 22 (1976), 149-160.

[ 7 ] R. C. T. Lee and C. L. Chang, Some properties of fuzzy logic, Information and Control, 19 (1971), 417-431.

[ 8 ] M. Takahashi, Many-valued logics of extended Gentzen style I, Sci. Rep. Tokyo Kyoiku Daigaku, 9 (1967), 271-292.

[9] — Many-valued logics of Gentzen style II-First order continuous logic, JSL, 35 (1970), 493-528.

[10] - Continuous $\lambda-\varepsilon$ logics, Ann. Jap. Assoc. Phil. Sci., 3 (1970), 205-215.

[11] G. Takeuti, Proof Theory, North-Holland Publ. Co., Amsterdam, 1975.

[12] L. A. Zadeh, Fuzzy sets, Information and Control, 8 (1965), 338-353.

[13] _ _ Quantitative fuzzy semantics, Information Science, 3 (1971), 159-176.

University of Tsukuba 